\title{
Destruction mechanisms and methods of laboratory autoclave tests of internal coatings of oil pipes
}

\author{
Pavel Yudin ${ }^{1, *}$, Sergei Petrov ${ }^{1}$, Andrey Maximuk ${ }^{1}$ and Zhanna Knyazeva ${ }^{1}$ \\ ${ }^{1}$ Limited Liability Company "Scientific and Production Center "Samara", 443022 Samara, Russian Federation
}

\begin{abstract}
The main mechanisms of destruction of internal anticorrosive polymer coatings (IACPC) of the inner surface of tubing and fielding pipes are review in the article. They are divide into decompression peeling, substrate destruction under the corrosion of and natural aging of the polymeric basis. A critical analysis of the existing standards for the testing of internal anticorrosive coatings was made. The carrying out test on these documents does not allow obtaining the destructions identical to those found during the exploitation of pipelines. Investigations on the selection of optimal regimes of autoclave tests and the methodology for conduct research are describe. A comparison for the results of laboratory and experimental field tests of the coatings presented.
\end{abstract}

\section{Introduction}

Annual metal losses due to corrosion account for up to $12 \%$ of the total mass of the metal-fund, which corresponds to a loss of up to $30 \%$ of annual metal production in the Russian Federation [1]. The problem of corrosion is especially acute in the oil industry, where, according to various data, the share of oil pipeline failures due to corrosion is 25 to $95 \%$ of the total number of accidents [2]. The costs of corrosion in 2012 in the US exceeded $\$ 1$ trillion that was about $6.2 \%$ of GDP $[3,4]$.

Only now in the extractive industry of the Russian Federation internal anticorrosive coatings are gaining increasing popularity for protection of the fielding pipes inner surface (usually epoxy and epoxy - Novolacs basis) despite the fact that the effectiveness of internal anticorrosion coatings was demonstrated 20 years ago [5]. All new capital construction takes place using this technology in most oil companies. Despite of the impressive economic effect an applicability of this technology for a long time has been limited to several aspects: the inability to conduct in-pipe diagnostics (due to the use of sleeves to protect the welded joint), the rapid process of corrosive destruction at the site of the defect, the inability to verify quality control and predict the resource.

Experience in the development of oil-fields in Western Siberia talk that the intensity of development by the corrosion damages to the internal surface of field pipelines is associated with an increase in the number of mechanical impurities in pumped product, changes in the ion composition of mining bedded water, flow regimes and significant bio-contamination of the oil-fields. This explains the growing need for corrosion protection methods for the internal surface of pipelines including the use of the IACPC. Along with the increase in the use of IACPC, there is a need to ensure and control the quality of the supplied products. Decision of this problem requires a complex approach, including the development of techniques for laboratory tests taking into account the specific exploitative conditions of the field pipelines. A complex approach to the test problem described in [6]. The authors analyzed a large number of causes of failures of the tubing and field pipelines and highlighted the main mechanisms of IACPC destruction during exploitation: decompression peeling of the coating, corrosive destruction of the substrate metal, aging of the polymeric basis, which confirms the conclusions made in work [7].

\section{Decompression peeling of the coating}

The essence of this destruction mechanism is the penetration of gas dissolved in the transported medium into the volume of the polymer through micropores and discontinuities, followed by the accumulation of gas in the pores or at the metal-coating interface. A multiple expansion of the compressed gas volume occurs with pressure drops. This accompanied by mechanical destruction of adhesive and cohesive bonds [8] and, as a result, the formation of swellings, exfoliations and cracking of the coating (Figure 1).

The main signs of decompression destruction include low (less than one year) time to failure $(\mathrm{TtF})$; large damage area; no corrosion products under coating; high power of deformation of the coating. This type of destruction is more typical for tubing than for oilfield communication pipelines.

\footnotetext{
${ }^{*}$ Corresponding author: office@npcsamara.ru.
} 

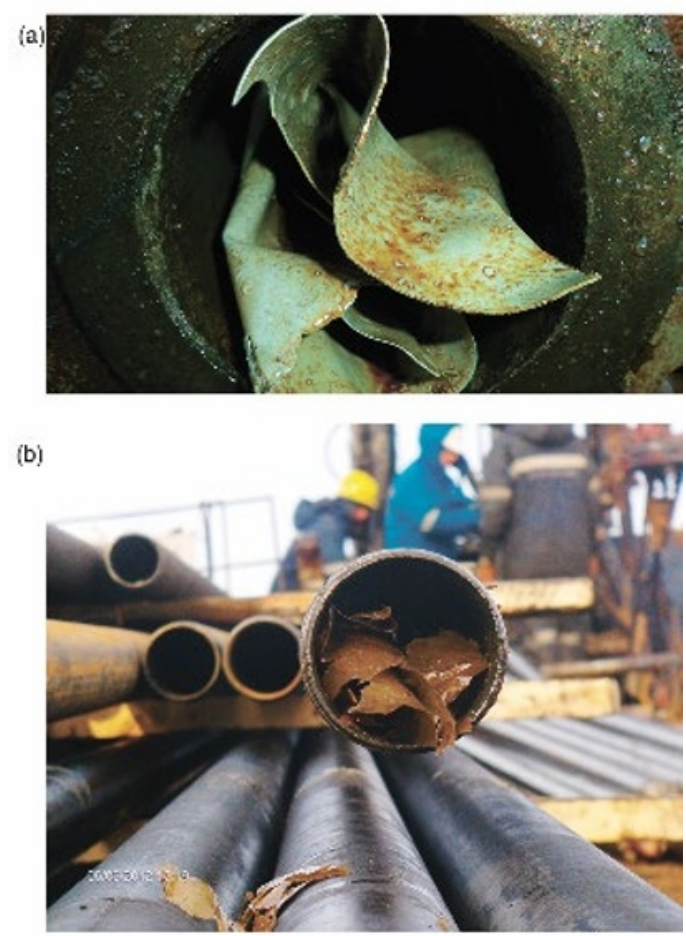

Fig. 1. Appearance of the coatings of the fielding pipe (a) and the tubing (b), destroyed by the mechanism of decompression peeling

\section{Corrosive destruction of substrate metal}

The physical meaning of this mechanism is the diffusion penetration of corrosion-active components of the medium through the coating to the "metal-coating" interface followed by chemical destruction of adhesive bonds and the formation of corrosion products of metal [9]. The stresses in the coating (that arise due to the difference in the density of the metal and the corrosion products) increase with the further development of corrosion destruction of the substrate metal. Any coating of the pipeline is more or less permeable to the components of the transported medium. The service life of the coating in this case is characterized by the gas (hydro) permeability of the polymeric basis of the coating and, primarily, is determined by its phase state, the flexibility of the polymeric chain and the nature of the intermolecular interactions. The destruction mechanism, as a rule, is characterized by a longer (more than three years) $\mathrm{TtF}$ in comparison with the mechanism of decompression peeling, brightly expressed foci of corrosion destruction of the pipe metal, local swellings and coating peelings (Figure 2) with following accelerated destruction of the pipe metal.

\section{Aging of polymeric basis}

The structure of the polymeric basis of the coating with long-term hydrothermal action undergoes a number of changes among which can be distinguished the degradation. The degradation of the basis occurs due to the reactions of polymeric chains with the components of the medium (hydrolysis, acidolysis, etc.), free radicals or ions. The glass transition temperature, mechanical and barrier properties of the coating with aging of the polymeric basis are reduced (the degradation of the polymeric basis is described for more details in [9]). The distinctive features of the destruction of the coating as a result of the aging of the polymeric basis include a significant (more than seven years) $\mathrm{TtF}$; reduction in strength properties of the material; local cracking of the coating without significant corrosion damage to the metal (Figure 3).

Simulation of all the above destruction mechanisms is possible with autoclave tests. There are several world standards to date that regulate this type of testing: API 5L2, API 5L7, NACE TM0185 and 09-SAMSS 091.

The test procedure consists, in general, in holding the prepared samples in a medium of a certain composition (both in the liquid and gas phases) with the determined temperature and pressure for a set time. The testing must carried out in a special vessel (autoclave) which made of corrosion-resistant steel and capable of withstanding the maximum operating pressure and temperature. Samples are maintained at a temperature of $93^{\circ} \mathrm{C}$ and a pressure of $14 \mathrm{MPa}$ for 16 hours in a $5 \%$ (by weight) aqueous solution of $\mathrm{NaCl}$ and in a gas mixture $0.5 \%$ (by volume) $\mathrm{H} 2 \mathrm{~S}, 5 \% \mathrm{CO} 2$ and $94.5 \% \mathrm{CH} 4$ with a subsequent rapid dropping of the pressure, when tested according to the recommendations of standards API 5L7-88. Standard NACE TM0185-2006 recommends that samples be held for a minimum of 16 hours at a determined temperature and pressure, after which the autoclave is cooled to $93^{\circ} \mathrm{C}$ and a slow pressure dropping occurs within 15-30 minutes. The most typical test medium for evaluation of the polymeric coatings should include hydrocarbon, water and gas phase. The composition of the gas phase itself and the pressure during the testing in this standard are not regulate. The test conditions in the 09-SAMSS 091 standard vary depending on the testing medium. Thus in a $5 \%$ by weight aqueous solution of $\mathrm{NaCl}$ and a gas mixture of $0.5 \%$ by volume of $\mathrm{H} 2 \mathrm{~S}, 5 \%$ of $\mathrm{CO} 2$ and $94.5 \%$ of $\mathrm{CH} 4$, and in a $100 \% \mathrm{CO} 2$ medium, testing are carried out at a temperature of $95^{\circ} \mathrm{C}$ and a pressure of 20 $\mathrm{MPa}$. The recommended testing temperature is $50^{\circ} \mathrm{C}$ a medium of $10 \% \mathrm{HCl}$. Steady state (temperature / pressure) should be maintained for a minimum of 18 hours after which the heating ceases. Then it is necessary to cool the device to $90^{\circ} \mathrm{C}$ and dropping the pressure for at least one and a maximum of three minutes. An aqueous medium saturated with calcium carbonate $\mathrm{CaCO} 3$ or a dry nitrogen gas medium at temperatures of $25 \pm 3^{\circ} \mathrm{C}$ and $25 \pm 6^{\circ} \mathrm{C}$ and pressures of $16.5 \pm 3.4 \mathrm{MPa}$ and $8.3 \pm 0.7 \mathrm{MPa}$, respectively, uses for testing API 5L2-2002 standard. Sampling at these parameters maintained within 24 hours with a subsequent rapid dropping of the pressure for a period of not more than five seconds.

A common drawback of all the presented standards is the fixed testing temperature. To protect against corrosion of oil pipeline, tubing, drill pipes, PVM manufacturers produce a large number of coatings for various exploitation conditions, and especially for 
different temperatures of transported fluid. Increasing the temperature often leads to a significant increase in the cost of the material, increasing its fragility and reducing the basic physico - mechanical properties. The dependence [10] (Figure 4) is known from which it can be seen that at a temperature above $110^{\circ} \mathrm{C}$ the rate of corrosion of the metal falls considerably, hence, the application of high-temperature coatings becomes inexpedient. The temperature of the transported fluid on most pipelines in the RF does not exceed $60{ }^{\circ} \mathrm{C}$ (less often $80^{\circ} \mathrm{C}$ ), therefore, testing the coatings at a temperature of $90-95{ }^{\circ} \mathrm{C}$ leads to excessive rejection. Testing at such temperatures will not allow us to make a correct conclusion about the applicability of the material in media with a temperature of $100{ }^{\circ} \mathrm{C}$ or higher on the other hand.

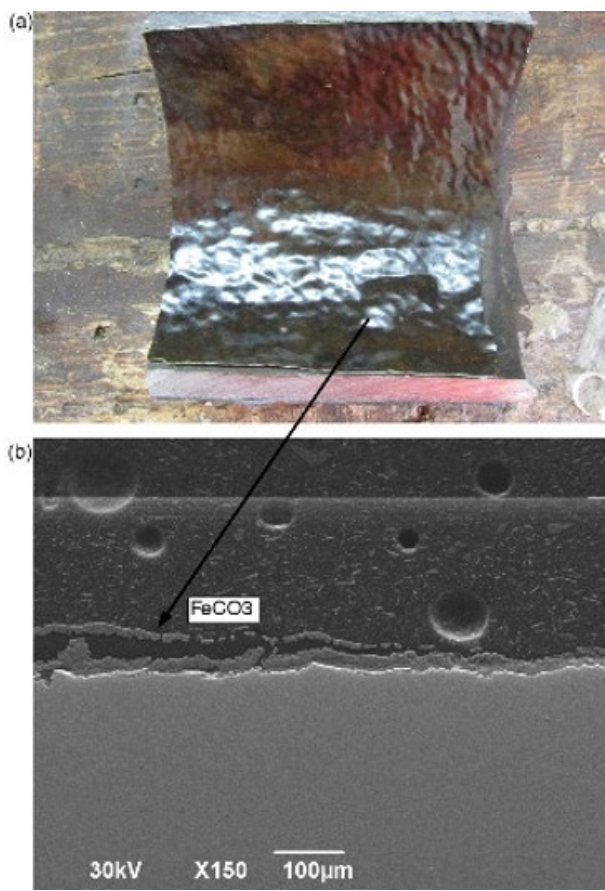

Fig. 2. Destruction of the pipeline coating by the corrosion mechanism of the pipe metal
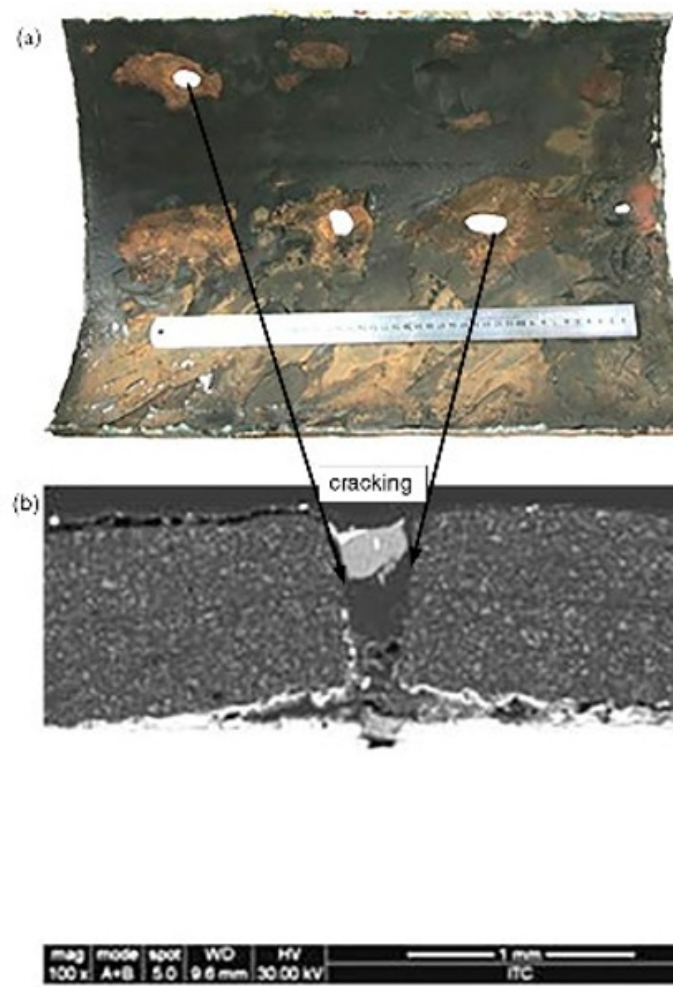

Fig.3. Destruction of the oilfield pipeline coating by the aging mechanism of the polymeric coating basis

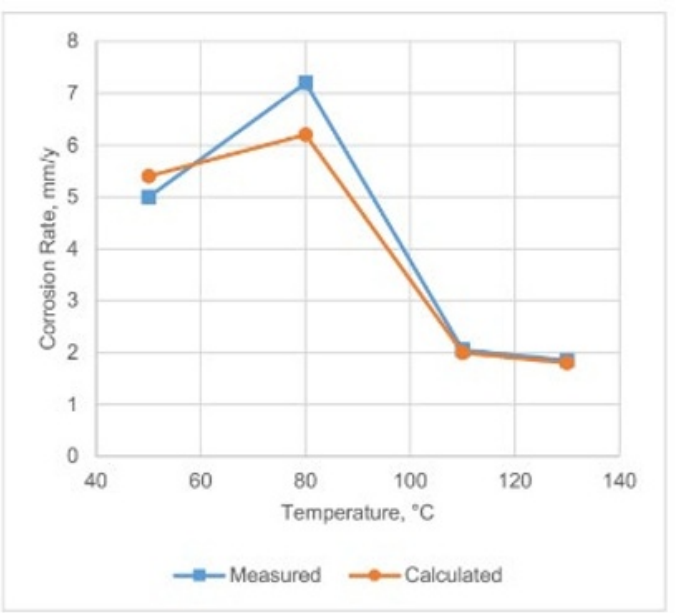

Fig.4. Dependence of corrosion rate of metal from temperature

Analysis the above normative documents shows that the testing carried out in different media. For example in API 5L2-2002, testing provided either in a dry nitrogen medium or in distilled water saturated with calcium carbonate. In the standards API 5L7-88, NACE TM0185-2006, 09-SAMSS 091, the testing are carried out in a multiphase medium (liquid + gas). Gas phase being either single-component (in the 09-SAMSS 091 standard it is $\mathrm{CO} 2$ or $\mathrm{N} 2$ ) or in multi-component (API $5 \mathrm{~L} 7-88$ provides for the use of a gas phase such as $0.5 \%$ $\mathrm{H} 2 \mathrm{~S}, 5 \% \mathrm{CO} 2,94.5 \% \mathrm{CH} 4)$. However, the presented standards do not regulate the ratio of the liquid and gas phases, which can lead to different gas concentrations in the liquid, where the basic diffusion processes take place directly. Obviously, for a small volume of gas in relation 
to water, its concentration in the liquid phase will be small, which will not allow us to fully assess the quality of the coatings. The testing results also affected by the character of the pressure dropping. A quick pressure dropping (decompression) is performed for example, in the standards API 5L7-88, API 5L2-2002 and 09SAMSS 091. Rapid decompression allows rapid assessment of the coating quality. Earlier [9] it was found that at a testing temperature exceeding the glass transition temperature of the polymeric coating basis, swellings and exfoliations are observed on the samples in almost $100 \%$ of cases. However, corrosion products at the "metal-coating" boundary do not have time to form. The maximum thickness of the layer of corrosion products can formed during the time of testing ( 1 day) will not exceed $30 \mu \mathrm{m}$, taking into account the fact that the rate of corrosion for low-alloy pipe steels under the described exploitative conditions does not exceed 10 $\mathrm{mm} /$ year [11]. Therefore, the testing conditions presented above make it possible to evaluate the resistance of coatings to rapid decompression and make an indirect assessment of the physico-mechanical properties, but they are do not evaluate their corrosion resistance, which should be one of the main criteria for assessing the applicability of coatings for the protection of oil pipelines. Testing on the API 5L2-2002 recommended carry out in an aqueous medium saturated with $\mathrm{CaCO} 3$, or in a dry nitrogen gas medium at room temperature. This technique intended for the investigation of gas pipelines and does not take into account the specifics of the exploitation of pipes intended for production and transportation of oil.

Execution tests on the above standards did not allow to obtain destruction to the coatings, identical to those identified during the examination of the causes of failure. The authors used coatings on epoxy and epoxy novolaks basis to protect the inner surface of pipes, both liquid and powder type (a total more than 50 different coating systems) to develop the methods of autoclave testing. The content of the gas and liquid phases, temperature, partial pressures of corrosive gases $(\mathrm{H} 2 \mathrm{~S}$ and $\mathrm{CO} 2$ ), total pressure in the autoclave, and the pressure drop rate varied during the experiments. Solution of $3 \%$ and $5 \% \mathrm{NaCl}$ in distilled water, crude oil, xylene + toluene mixture, $\mathrm{H} 2 \mathrm{~S}, \mathrm{CO} 2, \mathrm{~N} 2$ as gases were use as a liquid component. The following regularities were revealed:

I. A strong polar solvent and a gas highly soluble in the aqueous medium (whose molecules have a small diameter) should used as a liquid component of the medium. The experiment showed that at the analogous temperature, pressure and composition of the used gas (but the absence of a polar solvent) does not occur the appearance of swellings and the reduction of adhesion (Figure 5).

This relate to the fact that the sorption of gases by the polymer in the absence of moisture is not significant and it increases significantly with swelling of the coating [12]. The effect of the polar solvent is due to the ability of polar epoxy resins to swell in polar solvents, while nonpolar (or low polar) solvents demonstrate inertness. Swelling of the coating leads to an increase in the volume available for other media components, such as ions dissolved in liquid and gas molecules [12, 13]. Water is the most accessible solvent among polar liquids. Carbon dioxide and hydrogen sulfide have (among the gaseous components of the transported fluid) the greatest solubility in water, which is two orders of magnitude higher than the solubility of all other gases (hydrocarbons, nitrogen, etc.) [14]. Their sizes in addition are small enough to be able to efficiently sorb in micropores of the polymeric material. It should also be note that the presence of $\mathrm{NaCl}$ in water reduces the solubility of gases in water and the free pore volume (due to the sorption and filling of micropores $[12,13]$ ). Therefore the use of a $\mathrm{NaCl}$ solution reduces the probability of swelling and peeling at low pressures and temperatures. Nevertheless, the presence of the mineral component is necessary for modeling the test conditions for the coating which are the closest to the real ones. The concentration of sodium chloride in the solution is 5 wt.\% is optimal from the point of view of minimizing its effect on the solubility of gases and the sufficient mineralization and electrical conductivity of the medium [15].

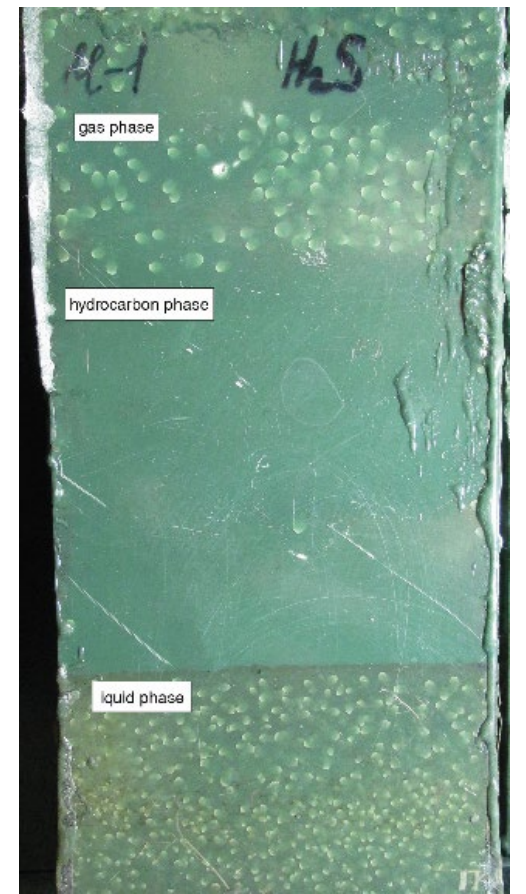

Fig. 5. Sample after a test in a multiphase medium

II. The minimum exposition time should be at least 4 hours for the appearance of the effect of decompression peeling. This time interval is due to the dissolution rate of corrosive gases and the time of gas diffusion through the coating. Obviously, the minimum duration of the establishment of equilibrium is different for different coatings that is determined by the diffusion rate of water and gas molecules in the polymeric material. In the framework of the research of the analyzed sample of coatings it was found that a $4 \mathrm{~h}$ aging time is sufficient for the appearance of a decompression exfoliation on all the samples studied. Nevertheless, it is necessary to use a longer time base to achieve the state of the system closest to equilibrium. Exposition for 24 hours is show 
the most optimal exposition and which considered as the standard duration of the test.

It was recommended to conduct the tests for 10 days to simulate the fracture mechanism associated with the corrosion of the substrate metal. Then the longer the exposure time, those the stronger the reduction in the adhesive strength of the coating. and the more pronounced the exposure time is the longer the exposure time is required to predict the life in specific exploitative media. Thus the more time it takes to predict the life in specific exploitation measures. However, exposure for 10 days is optimal to evaluate the quality and applicability of the coating at a given temperature because allows you to get results in a short time. Figure 6 shows the graph of adhesion change in coatings during laboratory autoclave testing and after field tests.

The time to failure in the course of field tests was 365 days for sample No.2, for which complete destruction of the adhesive bonds was observed after aging in the autoclave after 10 days (Figure 6). The nature of the change in adhesion during pilot-field tests and autoclave tests is almost identical for sample No.1 and No.3. Comparability of the curves in Fig. 6 make it possible to predict the resource, however, it is necessary to obtain more points on both graphs. These researches will described in subsequent works. A criterion was be adopted for the purposes of quality assessment during the 10-day autoclave coating test - a reduction in adhesion strength of not more than $50 \%$ of the original. This criterion has been adopted by most of the Russian oil companies, although there has been a trend towards tightening it (up to $30 \%$ ).

(a)

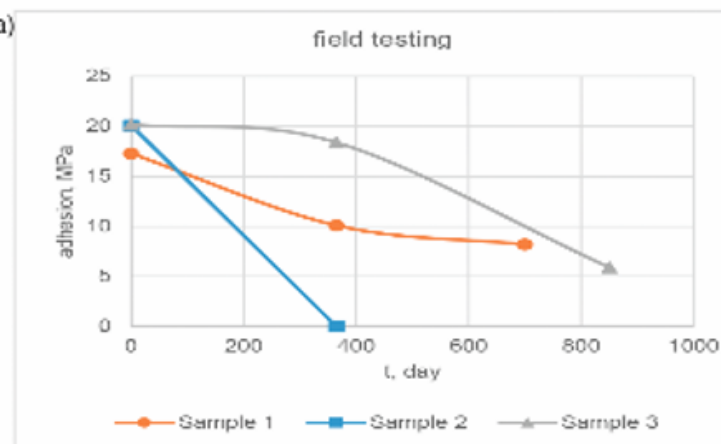

(b)

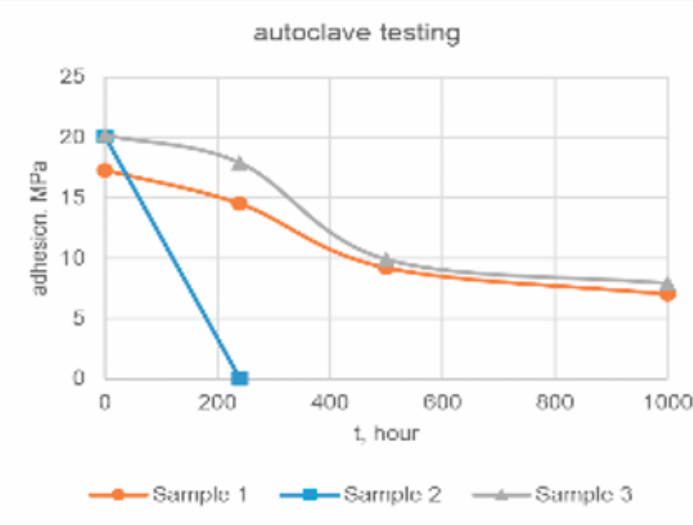

Fig. 6. Change in adhesion strength after field and autoclave testing: sample No.1 - powder epoxy coating grade PEP-585, sample No.2 - powder epoxy coating grade PEP-7150, sample No.3 - liquid epoxy coating

III. It is highly probable that a positive testing result can obtained at an autoclave temperature of $20-30^{\circ} \mathrm{C}$ below the glass transition temperature of the coating material (Figure 7). Presumably, the effect of reducing the glass transition temperature during testing is conditioned to the plasticization of the polymeric basis caused by water absorption of the coating. This research confirms the conclusions made in work [16].

The overpressure in the autoclave should be at least 3 $\mathrm{MPa}$ with respect to the atmospheric pressure. The solubility of gas in water, as well as the amount of gas absorbed by the polymer12, 13, is proportional to the partial pressure of the gas. It is necessary to accomplish the separation of the coating from the substrate or its cohesive destruction to provide such a pressure difference in the micropores of the coating and the external (with respect to the coating) medium to create internal stresses in the coating, the magnitude of which is sufficient to overcome of the adhesion and/or cohesion forces. The magnitude of this pressure is individual for each sample. Nevertheless, the standard value at which it is expedient to carry out the test for determining the resistance to decompression peeling, in this work a value of $5 \mathrm{MPa}$ is adopted. This pressure is optimal for the test, because, firstly, it corresponds to the maximum possible partial pressure of carbon dioxide gas in the oil pipelines, and secondly, this value with some margin is necessary and sufficient for the separation of any coating from the analyzed sample.

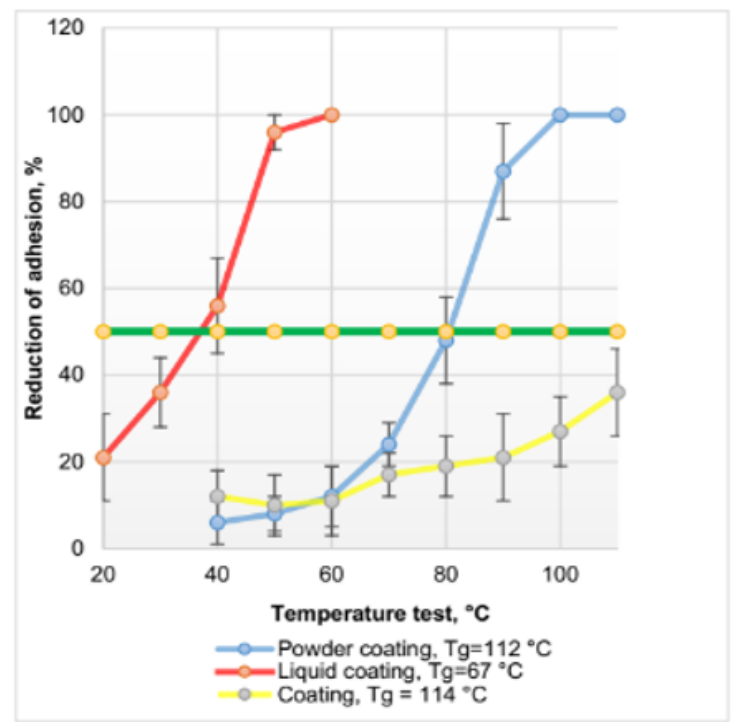

Fig. 7. Decrease of adhesion after autoclave tests at different temperatures glass

IV. The rate of pressure dropping during testing on the decompression peeling should be sufficient to ensure that the diffusion of the gas from the coating does not have time to occur and the gas pressure in the micropores creates internal stresses that lead to swelling of the coating. The guaranteed effect of decompression peeling comes at a pressure reset rate of at least $1 \mathrm{MPa} / \mathrm{s}$. On the contrary, this effect should be excluded if the corrosion resistance of coatings evaluated. It was 
experimentally established that in order to complete eliminate the effect of decompression peeling, the dropping rate should not exceed $1 \mathrm{MPa} / \mathrm{min}$.

The result of the work was the formulation of autoclave test method for determining the resistance to explosive decompression and evaluation of the corrosion resistance of the coating.

The present testing are carried out to determine changes in the appearance of the coating and adhesion to the steel after exposure to the test medium under pressure at an elevated temperature. Standard plates, specimen-witnesses or samples from products (hereinafter referred to as samples) of recommended sizes (150 mm long, 70 to $100 \mathrm{~mm}$ wide, at least $4 \mathrm{~mm}$ thick, at least 6 pieces thick) are used for the testing. All uncoated surfaces must protected by compositions that limit the access of the corrosive medium to the metal. The autoclave should have devices for measuring, monitoring and maintaining the specified temperature and pressure. A $5 \% \mathrm{NaCl}$ solution is prepared in distilled water. Samples are placed in an autoclave in such a way that the test surfaces do not contact each other and the walls of the vessel. The solution of sodium chloride is poured into the autoclave until the samples are completely immersed. The solution should occupy approximately $3 / 4$ of the volume of the vessel. The autoclave purged with nitrogen for at least 20 minutes. The autoclave is closed and carbon dioxide or hydrogen sulfide is fed into it until a predetermined pressure is reached at a temperature of $(20 \pm 5)^{\circ} \mathrm{C}$. The test medium maintained until the onset of the equilibrium state, but not less than 1 hour. Carbon dioxide or hydrogen sulphide supplied if necessary to the autoclave until a predetermined pressure reached. The test medium heated to a predetermined temperature and then nitrogen fed to the autoclave to a predetermined pressure. The time of the commencement of the testing record and conduct them for a predetermined time. Heating of the autoclave is switched off after the end of the tests and a pressure dropping is performed to simulate decompression peeling ( 24 hours) for no more than $5 \mathrm{~s}$, to simulate corrosion damage (240 hours) in not less than 10 minutes.

Testing of field pipelines coatings recommended carried out in mediums:

- modeling of decompression peeling the liquid phase $5 \% \mathrm{NaCl}$ solution in distilled water, gas phase of $\mathrm{CO} 2$ at a pressure $(5.0 \pm 0.5) \mathrm{MPa}$, exposition for 24 hours, dropping no more slowly than for $5 \mathrm{~s}$;

- modeling of corrosion destruction liquid phase $5 \%$ $\mathrm{NaCl}$ solution in distilled water, gas phase $(2.0 \pm 0.5)$ $\mathrm{MPa} \mathrm{CO} 2+(3.0 \pm 0.5) \mathrm{MPa} \mathrm{N} 2$ (for coatings of pipelines with dominant carbon-dioxide corrosion mechanism) and $(1,0 \pm 0,5) \mathrm{MPa} \mathrm{H} 2 \mathrm{~S}+(4,0 \pm 0,5) \mathrm{MPa}$ $\mathrm{N} 2$ (for coatings of pipelines with dominant hydrogensulphide corrosion mechanism), exposure for 240 hours, dropping no faster than 10 minutes.

Testing of tubing coatings recommended in mediums:

- modeling of decompression peeling the liquid phase $5 \% \mathrm{NaCl}$ solution in distilled water, gas phase of
$\mathrm{CO} 2$ at a pressure $(5.0 \pm 0.5) \mathrm{MPa}$, holding for 24 hours, dropping no more slowly than for $5 \mathrm{~s}$;

- modeling of corrosion destruction liquid phase $5 \%$ $\mathrm{NaCl}$ solution in distilled water, gas phase $(3,0 \pm 0,5)$ $\mathrm{MPa} \mathrm{CO} 2+(7,0 \pm 0,5) \mathrm{MPa} \mathrm{N} 2$ (for coatings of pipelines with dominant carbon-dioxide corrosion mechanism) and $(1,0 \pm 0,5) \mathrm{MPa} \mathrm{H} 2 \mathrm{~S}+(9,0 \pm 0,5) \mathrm{MPa}$ $\mathrm{N} 2$ (for coatings of pipelines with dominant hydrogensulphide corrosion mechanism), exposure for 240 hours, dropping no faster than 10 minutes.

The test temperature in all cases is regulated either by the manufacturer of the anticorrosion coating or by the consumer of the products based on the exploitative conditions.

Tests on the proposed methods have become widespread in the territory of the Russian Federation in many oil companies, which significantly increased the $\mathrm{TtF}$ of internal anticorrosive coatings. An example of the comparability of the results of field and laboratory testing is show in Figure 8

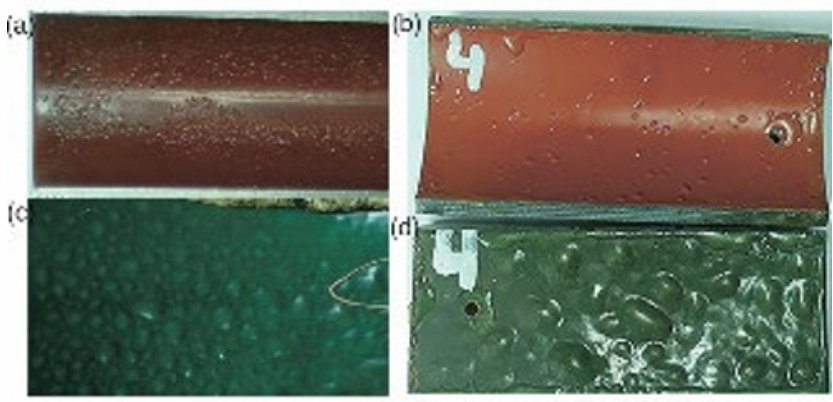

Fig. 8. Appearance of the internal anticorrosive epoxy coatings: a) Argof coating after exploitation for 1 year as a tubing; b) Argof coating after autoclave testing; c) TREPP TR coating after exploitation for 1 year as part of the oil collection collector (a synonym for the oil pipeline); d) TREPP TR coating after autoclave testing

\section{Conclusions}

- All destructions of internal polymeric anticorrosive coatings during exploitation can be divided into the following types: decompression peeling of the coating, corrosive destruction of the substrate metal, aging of the polymeric basis (failure caused by the violation of the coating technology in this work was not considered).

- The article presents a critical review of standards for autoclave testing API 5L7-88, API 5L2-2002, NACE TM0185-2006, 09-SAMSS 091. It is shown that carrying out testing using these methods does not allow obtaining destructions identical to commercial ones.

- It has been establish that a strong polar solvent (for example, an aqueous $\mathrm{NaCl}$ solution) must be used as the liquid component of the medium and a gas that is highly soluble in the aqueous medium whose molecules have a small diameter ( $\mathrm{CO} 2$ and $\mathrm{H} 2 \mathrm{~S}$ employed in the presented experiments)

- The minimum exposition time should be at least 4 hours for the appearance of the effect of decompression peeling. 
- The overpressure in the autoclave should be at least $3 \mathrm{MPa}$ with respect to the atmospheric pressure.

- The guaranteed effect of decompression peeling comes at a dropping pressure rate of at least $1 \mathrm{MPa} / \mathrm{s}$. It was experimentally established that in order to complete eliminate the effect of decompression peeling, the reset rate should not exceed $1 \mathrm{MPa} / \mathrm{min}$.

- A positive test result is observed for most polymeric coatings at an autoclave temperature of 20$30^{\circ} \mathrm{C}$ below the glass transition temperature of the coating material.

- An autoclave test procedure was proposed that showed good correlation with the results of a field testing and was taken as a base for the development of the national standard GOST R "Steel pipes and fittings for the oil industry. Protective coatings for internal surfaces. General specifications".

\section{References}

1. R. B. Faizov, Oil. Gaz. Ind. 3, 14 (2004).

2. S. A. Gurov, Increase the resource of safe operation of field pipelines based on the use of inhibitor protection, Ph. D. diss., Ufa Oil Tech. U., 24 (2003).

3. Chinedu I. Ossai, ISRN Corros. ID570143, 10 (2012).

4. Cao Shan, He Faqing, Gao Jinwei, Anti-Corros. Methods and Mater. 5, 465 (2017).

5. E. Y. Chen, T. Ahmed, Why Internally Coated Piping Is Used for the Worlds Largest Seawater Injection System (Annual Tech. Conf. and Ex. in New Orleans, 721-728, 1998).

6. J. Niglia, M. Marino, N. Hoijman, NACE Corros. Conf. 11035 (2011).

7. O. Knudsen, A. Bjorgum, A. Kvernbråten, NACE Corros. Conf., 10004 (2010).

8. E. Z. Yagubov, Development of principles for ensuring the structural reliability of oil and gas pipelines based on corrosion-resistant composite pipes, Ph. D. diss. Ukhta State Tech. U, 41 (2012).

9. E. V. Aleksandrov, P. E. Yudin, Zh. V. Knyazeva, Pipeline transport: theory and practice 3,60 (2015).

10. Zh. Yucheng, P. Xiaolu, Qa Shaopeng, Li Xin, Gao Kewei, Corros. Sci. 59, 186 (2012).

11. E. A. Borisenkova, A. G. Verevkin, T. A. Borisenkova, High technol. in eng. 4, 8 (2015).

12. A. Raytling, Permeability of the polymeric materials (RF: Chem., 1974).

13. A. A. Tager, Physico-chemistry of polymers (RF: Sci. world, 2007).

14. A. Yu. Namiot, Solubility of gases in water: Reference manual (RF: Nedra, 1991).

15. A.H. Mirzadjanzadeh, Technology and technology of oil production (RF: Nedra, 1986).

16. A. Husain, S.A. Bahar, J. Chakkamalayath, A. Vikreman, A. Ghamdi, T. Kamshad, R.S. Siriki, Eng. Failire Anal. 56, 375-383 (2015). 